\title{
PERSISTENCE AND EXTINCTION IN A PREDATOR-PREY MODEL CONSISTING OF NINE PREY GENOTYPES
}

\author{
JOSEPH W.-H. SO ${ }^{1}$
}

(Received I November 1988; revised 27 December 1988)

\begin{abstract}
A predator-prey model in which the prey population is subdivided into nine genotypes corresponding to a two-locus, two-allele problem is considered. Sufficient conditions are given which lead to extinction of all the prey allele types except one, as well as conditions which guarantee the persistence of all the allele types.
\end{abstract}

\section{Introduction}

In Freedman and Waltman [5, 6] and Freedman, So and Waltman [7], the authors considered a predator-prey model in which the prey population consists of three genotypes corresponding to a one-locus, two-allele problem. In this paper, we extend that model to the case when the prey population is subdivided into nine genotypes corresponding to a two-locus, two-allelle problem. Let $A$ and $a$ denote the two allele types at the first (prey) locus and let $B$ and $b$ denote the two allele types at the second locus. Since we shall not distinguish between the genotypes $A B / a b$ and $A b / a B$ (they will be combined and denoted by $A a B a$ ), we denote the number of prey of the genotype $A A B B$ (resp. $A A B b, A A b b, A a B B, A a B b, A a b b, a a B B, a a B b, a a b b$ ) by $x_{11}$ (resp. $x_{12}, x_{13}, x_{21}, x_{22}, x_{23}, x_{31}, x_{32}, x_{33}$ ). Also, let $y$ denote the number of

\footnotetext{
${ }^{1}$ Department of Mathematics, University of Alberta, Edmonton, Alberta, Canada T6G26

(C) Copyright Australian Mathematical Society 1990, Serial-fee code 0334-2700/90
} 
predators. The model we wish to consider is of the form:

$$
\begin{aligned}
& \dot{x}_{11}=\left(u_{1}^{2} / x^{2}\right) B(x)-\left(x_{11} / x\right)\left[D(x)+y P_{11}(x)\right] \\
& \dot{x}_{12}=\left(2 u_{1} u_{2} / x_{2}\right) B(x)-\left(x_{12} / x\right)\left[D(x)+y P_{12}(x)\right] \\
& \dot{x}_{13}=\left(u_{2}^{2} / x^{2}\right) B(x)-\left(x_{13} / x\right)\left[D(x)+y P_{13}(x)\right] \\
& \dot{x}_{21}=\left(2 u_{1} u_{3} / x^{2}\right) B(x)-\left(x_{21} / x\right)\left[D(x)+y P_{21}(x)\right] \\
& \dot{x}_{22}=\left(2\left(u_{1} u_{4}+u_{2} u_{3}\right) / x_{2}\right) B(x)-\left(x_{22} / x\right)\left[D(x)+y P_{22}(x)\right] \\
& \dot{x}_{23}=\left(2 u_{2} u_{4} / x^{2}\right) B(x)-\left(x_{23} / x\right)\left[D(x)+y P_{23}(x)\right] \\
& \dot{x}_{31}=\left(u_{3}^{2} / x^{2}\right) B(x)-\left(x_{31} / x\right)\left[D(x)+y P_{31}(x)\right] \\
& \dot{x}_{32}=\left(2 u_{3} u_{4} / x^{2}\right) B(x)-\left(x_{32} / x\right)\left[D(x)+y P_{32}(x)\right] \\
& \dot{x}_{33}=\left(u_{4}^{2} / x^{2}\right) B(x)-\left(x_{33} / x\right)\left[D(x)+y P_{33}(x)\right] \\
& \dot{y}=y\left[-s+k \sum_{i, j=1}^{3} \frac{x_{i j}}{x} P_{i j}(x)\right] \\
& x_{i j}(0) \geq 0 \quad(i, j=1,2,3), \quad y(0) \geq 0,
\end{aligned}
$$

where ${ }^{\cdot}=\frac{d}{d t}$ and $s, k>0$. Here $u_{1}$ (resp. $u_{2}, u_{3}$ and $u_{4}$ ) is the number of prey of the gamete type $A B$ (resp. $A b, a B$ and $a b$ ); that is,

$$
\begin{aligned}
& u_{1}=x_{11}+x_{12} / 2+x_{21} / 2+x_{22} / 4 \\
& u_{2}=x_{13}+x_{12} / 2+x_{23} / 2+x_{22} / 4 \\
& u_{3}=x_{31}+x_{21} / 2+x_{32} / 2+x_{22} / 4 \\
& u_{4}=x_{33}+x_{23} / 2+x_{32} / 2+x_{22} / 4,
\end{aligned}
$$

and $x$ is the total prey population, that is:

$$
x=\sum_{i, j=1}^{3} x_{i j}=\sum_{i=1}^{4} u_{i} .
$$

$B(x) / x$ (resp. $D(x) / x$ ) is the natural, intrinsic birth (resp. death) rate of the entire prey population. The constant $s$ is the death rate of the predator in the absence of prey. $P_{i j}(x)(i, j=1,2,3)$ is the predator functional response on the prey genotype $x_{i j}$. The constant $k$ is the conversion factor from prey biomass to predator biomass. $B(x), D(x)$, and $P_{i j}(x)(i, j=1,2,3)$ are assumed to satisfy (H1)-(H6) of So and Freedman [10], namely,

(H1) $B, D, P_{i j}:[0, \infty) \rightarrow[0, \infty)(i, j=1,2,3)$ are smooth $\left(C^{2}\right)$;

(H2) $B(0)=D(0)=P_{i j}(0)(i, j=1,2,3)$;

(H3) $B^{\prime}(x)>0, D^{\prime}(x)>0, P_{i j}^{\prime}(x)>0(i, j=1,2,3)$ for all $x \in[0, \infty)$;

(H4) $B^{\prime}(0)>D^{\prime}(0)$;

(H5) there exists a unique $K>0$ (carrying capacity of prey) such that $B(K)=$ $D(K)$; and,

(H6) $B^{\prime}(K)<D^{\prime}(K)$ where ${ }^{\prime}=d / d x$. 
The derivation of (1.1) is similar to that given in So [9]. Let $f$ be the common fertility of the nine prey genotypes. The total number of gametes in the gamete pool is given by $f u_{1}+f u_{2}+f u_{3}+f u_{4}=f x$. By the assumption of random union of gametes, the proportion of $A A B B$ genotypes produced is $\left(f u_{1} / f x\right)\left(f u_{1} / f x\right)=u_{1}^{2} / x^{2}$. This accounts for the coefficients of $B(x)$ in the first equation of (1.1). The other coefficients are derived similarly.

The main purpose of this paper is to obtain conditions under which system (1.1) exhibits uniform persistence (that is, there exists $\eta>0$ such that $\lim \inf _{t \rightarrow+\infty} x_{i j}(t) \geq \eta(i, j=1,2,3)$ and $\liminf _{t \rightarrow+\infty} \geq \eta$ for all solutions $\left(x_{11}(t), \ldots, x_{33}(t), y(t)\right)$ of $(1.1)$ with $x_{i j}(0)>0(i, j=1,2,3)$ and $\left.y(0)>0\right)$, as well as conditions which lead to the extinction of all the prey gamete types except one. In the process, we show that if the predator is absent then the prey genotypes converge to Hardy-Weinberg proportions. Also a comparison between the two-locus model (1.1) and the previously studied one-locus model will be discussed.

The remainder of the paper is organized as follows. In the next section we introduce the necessary notations and summarize some elementary facts about (1.1). In Section 3, the case when the predator is absent will be considered. Section 4 deals with the extinction cases, while the persistent cases are considered in Section 5. We finish with a discussion and comparison of our results for the two-locus model (1.1) with those for the one-locus model in Section 6.

\section{Preliminaries}

We first introduce some notations which will be useful later. Let $\mathbf{R}_{+}^{n}=$ $\left\{\left(z_{1}, \ldots, z_{n}\right) \in \mathbb{R}^{n}: z_{i} \geq 0(i=1, \ldots, n)\right\}$ denote the non-negative cone in $\mathbb{R}^{n}$. For a set $S \subset \mathbb{R}^{n}, c l(S)$ denotes it closure, $\stackrel{\circ}{S}$ denotes its interior and $\partial(S)$ denotes it boundary. In particular, $\stackrel{\circ}{*}_{+}^{n}$ denotes the positive cone in $\mathbb{R}^{n}$. Let $H_{z_{i}}$ denote the non-negative $z_{i}$ axis in $\mathbf{R}^{n}$, i.e., $H_{z_{i}}=\left\{\left(0, \ldots, 0, z_{i}, 0, \ldots, 0\right) \in\right.$ $\left.\mathbf{R}^{n}: z_{i} \geq 0\right\}$. Similarly, $H_{z_{i}, z_{j}}$ denotes the cone spanned by the non-negative $z_{i}$ and $z_{j}$ axes in $R^{n}$, i.e.,

$$
H_{z_{i}, z_{j}}=\left\{\left(0, \ldots, 0, z_{i}, 0, \ldots, 0, z_{\jmath}, 0, \ldots, 0\right) \in \mathbf{R}^{n}: z_{i}, z_{\jmath} \geq 0\right\},
$$

etc.

System (1.1) is not defined when $(X, y) \equiv\left(x_{11}, \ldots, x_{33}, y\right)=(0, \ldots, 0, y)$. When $(X, y)=(0, \ldots, 0, y)$, we define $(1.1)$ to be

$$
\dot{x}_{i j}=0, \quad \dot{y}=-s y .
$$


It is easily seen that with the extended definition (2.1), the right hand side (RHS) of (1.1) is locally Lipschitz on $\mathbf{R}_{+}^{10}$ and is $C^{2}$ on $\stackrel{\circ}{+}_{+}^{10}$. Thus (1.1) defines a continuous semiflow on $\mathbf{R}_{+}^{10}$ which becomes a smooth $\left(C^{2}\right)$ local flow when restricted to $\stackrel{\mathrm{R}}{+}_{+}^{10}$. Moreover, it is $\mathscr{A}$-dissipative with

$$
\mathscr{A}=\left\{\left(x_{11}, \ldots, x_{j j}, y\right) \in \mathbb{8}_{+}^{10}: x \leq K \text { and } k x+y \leq \frac{k}{s}(M+s K)\right\},
$$

where $M=\max \{B(x)-D(x): x \in[0, K]\}$. That is, for all solutions $(X(t)$, $y(t)) \equiv\left(x_{11}(t), \ldots, x_{33}(t), y(t)\right)$ of $(1.1)$, dist $(\mathscr{A},(X(t), y(t))) \rightarrow 0$ as $t \rightarrow+\infty$ where dist $(\cdot, \cdot)$ denotes the Euclidean distance (cf. p. 472-474 of So and Freedman [10]). Hence, solutions of (1.1) are bounded for all positive time. Further, $H_{x_{11}}, H_{x_{13}}, H_{x_{31}}, H_{x_{33}}, H_{y}, H_{x_{11}, y}, H_{x_{13}, y}, H_{x_{31}, y}, H_{x_{33}, y}$ are invariant and $H_{x_{11}, x_{12}, x_{13}}, H_{x_{11}, x_{21}, x_{31}}, H_{x_{13}, x_{23}, x_{33}}, H_{x_{31}, x_{32}, x_{33}}, H_{x_{11}, x_{12}, x_{13}, y}, H_{x_{11}, x_{21}, x_{31}, y}$, $H_{x_{13}, x_{23}, x_{33}, y}, H_{x_{31}, x_{32}, x_{33}, y}$, and $H_{x_{11}, \ldots, x_{33}}$ are positively invariant. The vector field $F_{(1.1)}$ defined by the RHS of (1.1) points into $\stackrel{\circ}{+}_{+}^{10}$ on $\partial\left(\mathbb{R}_{+}^{10}\right) \backslash \nu$, where

$$
\begin{aligned}
\nu= & H_{x_{11}, x_{12}, x_{13}, y} \cup H_{x_{11}, x_{21}, x_{31}, y} \cup H_{x_{13}, x_{23}, x_{33}, y} \\
& \cup H_{x_{31}, x_{32}, x_{33}, y} \cup H_{x_{11}, \ldots, x_{33}} .
\end{aligned}
$$

Thus, no point in $\partial\left(\mathbb{R}_{+}^{10}\right) \backslash \nu$ can lie in the omega limit set of any point in $\stackrel{\circ}{+}_{+}^{10}$.

We will now list some of the equilibrium points of (1.1) and discuss their stability. More discussions will be given in later sections. By (2.1), $E_{0}=$ $(0, \ldots, 0,0)$ is an equilibrium point of $(1.1)$. System (1.1) when restricted to the invariant set $H_{x_{i}, y}(i, j=1,3)$ is the usual generalized Gauss predatorprey model (c.f. Chapter 4 of Freedman [4]). Thus $H_{x_{i}, y}(i, j=1,3)$ contains a positive equilibrium point if and only if $b_{i j}>0(i, j=1,3)$, where

$$
b_{i j}=-s+k P_{i j}(K) \quad(i, j=1,3) .
$$

Also, these equilibrium points are unique, by $(\mathrm{H} 3)$. Let us denote them by $E_{l}(i=1, \ldots, 4)$. That is, let $E_{1}=\left(\bar{x}_{11}, 0, \ldots, 0, \bar{y}_{11}\right), E_{2}=\left(0,0, \bar{x}_{13}, 0, \ldots\right.$, $\left.0, \bar{y}_{13}\right), E_{3}=\left(0, \ldots, 0, \bar{x}_{31}, 0,0, \bar{y}_{31}\right)$, and $E_{4}=\left(0, \ldots, 0, \bar{x}_{33}, \bar{y}_{33}\right)$ where $\bar{x}_{i j}$, $\bar{y}_{i j}>0(i, j=1,3)$. System (1.1) when restricted to the positively invariant set $H_{x_{11}, x_{21}, x_{31}, y}$ (resp. $H_{x_{11}, x_{12}, x_{13}, y}, H_{x_{13}, x_{23}, x_{33}, y}$, and $H_{x_{31}, x_{32}, x_{33}, y}$ ) is the one-locus, two-allele model, considered in Freedman and Waltman [5,6] and Freedman, So and Waltman [7], whose global dynamics is known when there is an ordering of the predator functional responses. In particular, we point out Theorem 3.1 of Freedman, So and Waltman [7], which will be used repeatedly in Sections 4 and 5. The positively invariant sets $H_{x_{11}, x_{21}, x_{31}, y}$, $H_{x_{11}, x_{12}, x_{13}, y}, H_{x_{13}, x_{23}, x_{33}, y}$, and $\stackrel{\circ}{H}_{x_{31}, x_{32}, x_{33}, y}$ may or may not contain equilibrium points. Some conditions are known which guarantee the existence or nonexistence of these equilibrium points (see Freeman, So and Waltman [7]). 
However, we will not use these criteria in what follows. Finally, it will be shown in Corollary 3.3 that $H_{x_{11}, \ldots, x_{33}}$ contains a two-dimensional set of equilibrium points.

$E_{0}$ is unstable and in fact we have the following proposition.

Proposition 2.1. For all $(\hat{X}, \hat{y}) \equiv\left(\hat{x}_{11}, \ldots, \hat{x}_{33}, \hat{y}\right) \in \stackrel{\circ}{\mathbf{R}}_{+}^{10}$, we have, $E_{0} \notin$ $\omega(\hat{X}, \hat{y})$, the omega limit set of the point $(\hat{X}, \hat{y})$.

Proof. First we observe that since the flow on $\stackrel{\circ}{H}_{y}$ is given by $\dot{y}=-s y$ whose negative orbits are all unbounded, $\left(0, \ldots, 0, y^{*}\right) \notin \omega(\hat{X}, \hat{y})$ for any $y^{*}>0$. Next, for $(X, y)$ near $E_{0}$, we have

$$
\dot{x} \geq B(x)-D(x)-y P(x) \geq k_{1} x \text { for some } k_{1}>0
$$

and

$$
\dot{y} \leq y(-s+k P(x)) \leq-k_{2} y \text { for some } k_{2}>0,
$$

where $P(x)=\max \left\{P_{i j}(x): 0 \leq x \leq K, i, j=1,2,3\right\}$. Therefore $\left\{E_{0}\right\}$ is an isolated invariant set and its stable set, $W^{s}\left(E_{0}\right)$, is $H_{y}$. According to Theorem 4.1 of Butler and Waltman [1], if $E_{0} \in \omega(\hat{X}, \hat{y})$ then $W^{s}\left(E_{0}\right) \cap$ $\omega(\hat{X}, \hat{y})$ contains a point other than $E_{0}$, which is a contradiction. (See also Lemma 1 of Freedman and So [10].)

TABLE 1. $M\left(E_{1}\right)$ is given by

\begin{tabular}{|c|c|c|c|c|c|c|c|c|c|c|} 
& $x_{11}$ & $y$ & $x_{12}$ & $x_{13}$ & $x_{21}$ & $x_{22}$ & $x_{23}$ & $x_{32}$ & $x_{32}$ & $x_{33}$ \\
\hline$x_{11}$ & $?$ & $-v e$ & $?$ & $?$ & $?$ & $?$ & $?$ & $?$ & $?$ & $?$ \\
\hline$y$ & $+v e$ & 0 & $?$ & $?$ & $?$ & $?$ & $?$ & $?$ & $?$ & $?$ \\
\hline$x_{12}$ & 0 & 0 & $?$ & $+v e$ & 0 & $+v e$ & $+v e$ & 0 & 0 & 0 \\
\hline$x_{13}$ & 0 & 0 & 0 & $-v e$ & 0 & 0 & 0 & 0 & 0 & 0 \\
\hline$x_{21}$ & 0 & 0 & 0 & 0 & $?$ & $+v e$ & 0 & $+v e$ & $+v e$ & 0 \\
\hline$x_{22}$ & 0 & 0 & 0 & 0 & 0 & $?$ & $+v e$ & 0 & $+v e$ & $+v e$ \\
\hline$x_{23}$ & 0 & 0 & 0 & 0 & 0 & 0 & $-v e$ & 0 & 0 & 0 \\
\hline$x_{31}$ & 0 & 0 & 0 & 0 & 0 & 0 & 0 & $-v e$ & 0 & 0 \\
\hline$x_{32}$ & 0 & 0 & 0 & 0 & 0 & 0 & 0 & 0 & $-v e$ & 0 \\
\hline$x_{33}$ & 0 & 0 & 0 & 0 & 0 & 0 & 0 & 0 & 0 & $-v e$ \\
\hline
\end{tabular}

where +ve (resp. $-v e, 0, ?)$ means that the entry is positive (resp. is negative, zero, has indeterminate sign). 
The stability of $E_{i}(i=1,2,3,4)$ is governed by the signs of the real parts of the eigenvalues of the variational matrix $M\left(E_{i}\right)(i=1,2,3,4)$ of $(1.1)$ evaluated at $E_{i}(i=1,2,3,4)$. For illustration we present $M\left(E_{1}\right)$ in Table 1. Note that $y$ is moved from the $10^{\text {th }}$ to the $2^{\text {nd }}$ position.

The nonzero entries of $M\left(E_{1}\right)$ are given by:

$$
\begin{aligned}
& M_{\mathrm{i} i \mathrm{i} i}\left(E_{\mathrm{i}}\right)=B^{\prime}\left(\bar{x}_{\mathrm{i} i}\right)-D^{\prime}\left(\bar{x}_{\mathrm{i}}\right)-\bar{y}_{\mathrm{ii}} P_{\mathrm{ii}}^{\prime}\left(\bar{x}_{\mathrm{i}}\right) \\
& M_{11, y}\left(E_{1}\right)=-P_{11}\left(\bar{x}_{11}\right)<0 \\
& M_{11,12}\left(E_{1}\right)=M_{11,21}\left(E_{1}\right)=B^{\prime}\left(\bar{x}_{11}\right)-D^{\prime}\left(\bar{x}_{11}\right)-\bar{y}_{11} P_{11}^{\prime}\left(\bar{x}_{11}\right) \\
& M_{11,22}\left(E_{1}\right)=-B\left(\bar{x}_{11}\right) /\left(2 \bar{x}_{11}\right)+B^{\prime}\left(\bar{x}_{11}\right)-D^{\prime}\left(\bar{x}_{11}\right)-\bar{y}_{11} P_{11}^{\prime}\left(\bar{x}_{11}\right) \\
& M_{11, i j}\left(E_{1}\right)=-B\left(\bar{x}_{11}\right) / \bar{x}_{11}+B^{\prime}\left(\bar{x}_{11}\right) D^{\prime}\left(\bar{x}_{11}\right)-\bar{y}_{11} P_{11}^{\prime}\left(\bar{x}_{11}\right) \\
& \text { for }(i, j)=(1,3),(2,3),(3,1),(3,2) \text { and }(3.3) \\
& M_{y, 11}\left(E_{1}\right)=k \bar{y}_{11} P_{11}^{\prime}\left(\bar{x}_{11}\right)>0 \\
& M_{y, i j}\left(E_{1}\right)=\left(k \bar{y}_{11} / \bar{x}_{11}\right)\left[\bar{x}_{11} P_{11}^{\prime}\left(\bar{x}_{11}\right)+P_{i j}\left(\bar{x}_{11}\right)-P_{11}\left(\bar{x}_{11}\right)\right] \text {, } \\
& \text { for }(i, j) \neq(1,1) \\
& M_{12,12}\left(E_{1}\right)=\frac{1}{\bar{x}_{11}}\left[B\left(\bar{x}_{11}\right)-D\left(\bar{x}_{11}\right)-\bar{y}_{11} P_{12}\left(\bar{x}_{11}\right)\right]=\frac{\bar{y}_{11}}{\bar{x}_{11}}\left[\frac{s}{k}-P_{12}\left(\bar{x}_{11}\right)\right] \\
& M_{12,13}\left(E_{1}\right)=B\left(\bar{x}_{11}\right) / \bar{x}_{11}>0 \\
& M_{12,22}\left(E_{1}\right)=B\left(\bar{x}_{11}\right) /\left(2 \bar{x}_{11}\right)>0 \\
& M_{12,23}\left(E_{1}\right)=B\left(\bar{x}_{11}\right) / \bar{x}_{11}>0 \\
& M_{13,13}\left(E_{1}\right)=-\left[D\left(\bar{x}_{11}\right)+\bar{y}_{11} P_{13}\left(\bar{x}_{11}\right)\right] / \bar{x}_{11}<0 \\
& M_{21,21}\left(E_{1}\right)=\frac{1}{\bar{x}_{11}}\left[B\left(\bar{x}_{11}\right)-D\left(\bar{x}_{11}\right)-\bar{y}_{11} P_{21}\left(\bar{x}_{11}\right)\right]=\frac{\bar{y}_{11}}{\bar{x}_{11}}\left[\frac{s}{k}-P_{21}\left(\bar{x}_{11}\right)\right] \\
& M_{21,22}\left(E_{1}\right)=B\left(\bar{x}_{11}\right) /\left(2 \bar{x}_{11}\right)>0 \\
& M_{21,31}\left(E_{1}\right)=B\left(\bar{x}_{11}\right) / \bar{x}_{11}>0 \\
& M_{21,32}\left(E_{1}\right)=B\left(\bar{x}_{11}\right) / \bar{x}_{11}>0 \\
& M_{22,22}\left(E_{1}\right)=\frac{1}{\bar{x}_{11}}\left[\frac{1}{2} B\left(\bar{x}_{11}\right)-D\left(\bar{x}_{11}\right)-\bar{y}_{11} P_{22}\left(\bar{x}_{11}\right)\right] \\
& =\frac{1}{\bar{x}_{11}}\left[\bar{y}_{11}\left(\frac{s}{k}-P_{22}\left(\bar{x}_{11}\right)\right)-\frac{1}{2} B\left(\bar{x}_{11}\right)\right] \\
& M_{22,23}\left(E_{1}\right)=B\left(\bar{x}_{11}\right) / \bar{x}_{11}>0 \\
& M_{22,32}\left(E_{1}\right)=B\left(\bar{x}_{11}\right) / \bar{x}_{11}>0 \\
& M_{22,23}\left(E_{1}\right)=2 B\left(\bar{x}_{11}\right) / \bar{x}_{11}>0 \\
& M_{23,23}\left(E_{1}\right)=-\left[D\left(\bar{x}_{11}\right)+\bar{y}_{11} P_{23}\left(\bar{x}_{11}\right)\right] / \bar{x}_{11}<0 \\
& M_{31,31}\left(E_{1}\right)=-\left[D\left(\bar{x}_{11}\right)+\bar{y}_{11} P_{31}\left(\bar{x}_{11}\right)\right] / \bar{x}_{11}<0 \\
& M_{32,32}\left(E_{1}\right)=-\left[D\left(\bar{x}_{11}\right)+\bar{y}_{11} P_{32}\left(\bar{x}_{11}\right)\right] / \bar{x}_{11}<0 \\
& M_{33,33}\left(E_{1}\right)=-\left[D\left(\bar{x}_{11}\right)+\bar{y}_{11} P_{33}\left(\bar{x}_{11}\right)\right] / \bar{x}_{11}<0 \text {. }
\end{aligned}
$$


Therefore $M\left(E_{1}\right)$ has five negative eigenvalues: $M_{13,13}\left(E_{1}\right), M_{23,23}\left(E_{1}\right)$, $M_{31,31}\left(E_{1}\right), M_{32,32}\left(E_{1}\right), M_{33,33}\left(E_{1}\right)$; three real eigenvalues with indeterminate signs: $M_{12,12}\left(E_{1}\right), M_{21,21}\left(E_{1}\right), M_{22,22}\left(E_{1}\right)$; and the sign of the real parts of the two remaining eigenvalues (corresponding to eigenvectors lying on the $x_{11}-y$ plane) is the same as that of the sign of $M_{11,11}\left(E_{1}\right)$.

In order to apply Theorem 3.1 and Theorem 4.1 of Butler and Waltman [1] to show persistence in the latter sections, we need to know the stable set of a boundary equilibrium point, for example, the $E_{i}$ 's. The following proposition is a very useful result, which allows us to show that the intersection of the stable manifold of a hyperbolic boundary equilibrium point with the positive cone $\stackrel{R}{+}_{+}^{n}$, is empty, by knowing only one unstable eigen-direction.

Proposition 2.2. Consider $\dot{x}=f(x)\left(x \in \mathbf{R}^{n}\right)$. Assume that $f$ is $C^{2}$ and that $\mathbf{R}_{+}^{n}$ is positively invariant. Let $E \in \partial\left(\mathbf{R}_{+}^{n}\right)$ be a hyperbolic equilibrium point. Denote the stable (resp. unstable) manifold of $E$ by $W^{s}(E)\left(\right.$ resp. $\left.W^{u}(E)\right)$. If $W^{u}(E) \cap\left(\mathbb{R}^{n} \backslash \mathbb{R}_{+}^{n}\right) \neq \phi$, then $W^{s}(E) \cap \stackrel{\circ}{R}_{+}^{n}=\phi$.

Proof. Suppose not; that is, suppose $W^{s}(E) \cap \stackrel{\circ}{R}_{+}^{n} \neq \phi$. If we can show that for fixed $p_{1} \in W^{s}(E)$ and $p_{2} \in W^{u}(E)$ where $p_{1}, p_{2} \neq E$ and for arbitrary $\varepsilon>0$, there exists a point $p$ and a time $t>0$ such that $p$ is $\varepsilon$ close to $p_{1}$ and $\pi(p, t)$ is $\varepsilon$ close to $p_{2}$, then this will contradict the positive invariance of $\mathbb{R}_{+}^{n}$. Since this can be reduced to a local question, by Hartman-Grobman's theorem, it suffices to establish the following.

Consider the system $\dot{x}=A x, \dot{y}=B y$ where $x \in \mathbb{R}^{m}, y \in \mathbb{R}^{n}$, and all the eigenvalues of $A$ have negative real parts and all the eigenvalues of $B$ have positive real parts. Fix $p_{1}=\left(x^{*}, 0\right)$ and $p_{2}=\left(0, y^{*}\right) \in \mathbf{R}^{m+n}$ with $x^{*} \neq 0$ and $y^{*} \neq 0$. Then there exists $\left(x_{N}, y_{N}\right) \in \mathbf{R}^{m+n}$ and $t_{N}>0$ such that $\left(x_{N}, y_{N}\right) \rightarrow\left(x^{*}, 0\right)$ and $\pi\left(\left(x_{N}, y_{N}\right), t_{N}\right) \rightarrow\left(0, y^{*}\right)$ as $N \rightarrow \infty$.

For a proof of the above statement, let $t_{N}=N, x_{N}=x^{*}$ and $y_{N}=e^{-t_{N} B} y^{*}$. Then clearly $x_{N} \rightarrow x^{*}$ and $y_{N} \rightarrow 0$ as $N \rightarrow \infty$, since all eigenvalues of $B$ have positive real parts. Furthermore, we have,

$$
\pi\left(\left(x_{N}, y_{N}\right), t_{N}\right)=\left(e^{t_{N} A} x_{N}, e^{t_{N} B} y_{N}\right)=\left(e^{t_{N} A} x^{*}, y^{*}\right) \rightarrow\left(0, y^{*}\right)
$$

as $N \rightarrow \infty$.

\section{The case of no predators}

In this section we shall consider the global dynamics of (1.1) when the predator $y$ is absent. For the sake of future reference, let us refer to the 
resulting system with nine equations as (NP). As in Section 2, we define the RHS of (NP) as the zero vector $(0, \ldots, 0) \in \mathbf{R}^{9}$ when $X \equiv\left(x_{11}, \ldots, x_{33}\right)=$ $(0, \ldots, 0)$. Then the RHS of (NP) will be a locally Lipschitz vector field $F_{(\mathrm{NP})}$ and (NP) defines a continuous semi-flow on $R_{+}^{9}$ which becomes a smooth $\left(C^{2}\right)$ local flow when restricted to $\stackrel{\circ}{\mathbf{R}}_{+}^{9}$. Since

$$
\dot{x}=\vec{B}(x)-D(x),
$$

we have

$$
\lim _{t \rightarrow \infty} x(t)=K \quad \text { for all } x(0)>0 .
$$

Let $\sigma$ denote the simplex

$$
\sigma=\left\{X \equiv\left(x_{11}, \ldots, x_{33}\right) \in \mathbb{R}_{+}^{9}: \sum_{l, j=1}^{3} x_{l j}=K\right\} .
$$

Then $\sigma$ is positively invariant and is globally stable over $\mathbb{R}_{+}^{9} \backslash\{(0, \ldots, 0)\}$. $(0, \ldots, 0)$ is a repelling equilibrium point. $H_{x_{11}}, H_{x_{13}}, H_{x_{31}}$ and $H_{x_{33}}$ are invariant and $H_{x_{11}, x_{12}, x_{13}}, H_{x_{11}, x_{21}, x_{31}}, H_{x_{13}, x_{23}, x_{33}}$ and $H_{x_{31}, x_{32}, x_{33}}$ are positively invariant. The vector field $F_{(\mathrm{NP})}$ points into $\mathbb{R}_{+}^{9}$ on $\partial\left(\mathbb{R}_{+}^{9}\right) \backslash\left(H_{x_{11}, x_{12}, x_{13}} \cup H_{x_{11}, x_{21}, x_{31}} \cup\right.$ $H_{x_{13}, x_{23}, x_{33}} \cup H_{x_{31}, x_{32}, x_{33}}$ ).

Clearly, by (3.2), all equilibrium points of (NP) other than $(0, \ldots, 0)$ lie in $\sigma$. The following proposition and its first corollary describe the set of equilibrium points of (NP), which will be referred to as the Hardy-Weinberg manifold of (NP).

Proposition 3.1. Define $H: \stackrel{\circ}{\mathbf{R}}_{+}^{2} \rightarrow \sigma$ by

$$
H\left(c_{1}, c_{2}\right)=\left(H_{11}\left(c_{1}, c_{2}\right), \ldots, H_{33}\left(c_{1}, c_{2}\right)\right) \text { for }\left(c_{1}, c_{2}\right) \in \stackrel{\circ}{\mathbf{R}}_{+}^{2}
$$

where

$$
\begin{aligned}
& H_{11}\left(c_{1}, c_{2}\right)=c_{1}^{2} c_{2}^{2} K /\left[\left(1+c_{1}\right)^{2}\left(1+c_{2}\right)^{2}\right] \\
& H_{12}\left(c_{1}, c_{2}\right)=2 c_{1}^{2} c_{2} K /\left[\left(1+c_{1}\right)^{2}\left(1+c_{2}\right)^{2}\right] \\
& H_{13}\left(c_{1}, c_{2}\right)=c_{1}^{2} K /\left[\left(1+c_{1}\right)^{2}\left(1+c_{2}\right)^{2}\right] \\
& H_{21}\left(c_{1}, c_{2}\right)=2 c_{1} c_{2}^{2} K /\left[\left(1+c_{1}\right)^{2}\left(1+c_{2}\right)^{2}\right] \\
& H_{22}\left(c_{1}, c_{2}\right)=4 c_{1} c_{2} K /\left[\left(1+c_{1}\right)^{2}\left(1+c_{2}\right)^{2}\right] \\
& H_{23}\left(c_{1}, c_{2}\right)=2 c_{1} K /\left[\left(1+c_{1}\right)^{2}\left(1+c_{2}\right)^{2}\right] \\
& H_{31}\left(c_{1}, c_{2}\right)=c_{2}^{2} K /\left[\left(1+c_{1}\right)^{2}\left(1+c_{2}\right)^{2}\right] \\
& H_{32}\left(c_{1}, c_{2}\right)=2 c_{2} K /\left[\left(1+c_{1}\right)^{2}\left(1+c_{2}\right)^{2}\right] \\
& H_{33}\left(c_{1}, c_{2}\right)=K /\left[\left(1+c_{1}\right)^{2}\left(1+c_{2}\right)^{2}\right] .
\end{aligned}
$$


Then $H\left(\stackrel{\circ}{\mathbf{R}}_{+}^{2}\right)$, the image of $\stackrel{\circ}{\mathbf{R}}_{+}^{2}$ under $H$, is the set of all equilibrium points of (NP) in $\stackrel{\circ}{\mathbf{R}}_{+}^{9}$.

Proof. First we show that all points of the form $H\left(c_{1}, c_{2}\right)$ where $\left(c_{1}, c_{2}\right) \in \stackrel{\circ}{R}_{+}^{2}$ are equilibrium points of (NP). Let $H\left(c_{1}, c_{2}\right)=X^{*} \equiv\left(x_{11}^{*}, \ldots, x_{33}^{*}\right)$. Clearly $X^{*} \in \stackrel{\circ}{\mathrm{R}}_{+}^{9}$. Define, as in (1.2),

$$
\begin{aligned}
& u_{1}^{*}=x_{11}^{*}+x_{12}^{*} / 2+x_{21}^{*} / 2+x_{22}^{*} / 4 \\
& u_{2}^{*}=x_{13}^{*}+x_{12}^{*} / 2+x_{23}^{*} / 2+x_{22}^{*} / 4 \\
& u_{3}^{*}=x_{31}^{*}+x_{21}^{*} / 2+x_{32}^{*} / 2+x_{22}^{*} / 4 \\
& u_{4}^{*}=x_{33}^{*}+x_{23}^{*} / 2+x_{32}^{*} / 2+x_{22}^{*} / 4
\end{aligned}
$$

and

$$
x^{*}=x_{11}^{*}+\cdots+x_{33}^{*}
$$

Then

$$
\begin{array}{cc}
u_{1}^{*}=c_{1} c_{2} K /\left[\left(1+c_{1}\right)\left(1+c_{2}\right)\right], & u_{2}^{*}=c_{1} K /\left[\left(1+c_{1}\right)\left(1+c_{2}\right)\right], \\
u_{3}^{*}=c_{2} K /\left[\left(1+c_{1}\right)\left(1+c_{2}\right)\right], & u_{4}^{*}=K /\left[\left(1+c_{1}\right)\left(1+c_{2}\right)\right]
\end{array}
$$

and $x^{*}=K$. Therefore the first component $F_{(\mathrm{NP}) 11}$ of the RHS of (NP) when evaluated at $X^{*}$ is given by

$$
\left(u_{1}^{* 2} / x^{* 2}\right) B\left(x^{*}\right)-\left(x_{11}^{*} / x^{*}\right) D\left(x^{*}\right)=\left(u_{1}^{* 2}-x_{11}^{*} K\right) B(K) / K^{2}=0 .
$$

Similarly, one shows that the other components of the RHS of (NP) are all equal to 0 and hence $X^{*}$ is an equilibrium point.

Next, we show that every interior equilibrium point of (NP) is of the form $H\left(c_{1}, c_{2}\right)$ for some $\left(c_{1}, c_{2}\right) \in \stackrel{\circ}{R}_{+}^{2}$. Let $X^{*}=\left(x_{11}^{*}, \ldots, x_{33}^{*}\right)$ be an equilibrium point (NP) with $x_{i j}^{*}>0(i, j=1,2,3)$. Define $u_{1}^{*}, u_{2}^{*}, u_{3}^{*}, u_{4}^{*}$ and $x^{*}$ as in (3.5). Clearly $x^{*}=K$. Also define

$$
d_{i j}=x_{i j}^{*} / x_{33}^{*} \quad(i, j=1,2,3)
$$

and

$$
r_{i}=u_{i}^{*} / x_{33}^{*} \quad(i=1, \ldots, 4)
$$


Then

$$
\begin{aligned}
r_{1}^{2} x_{33}^{*} & =d_{11} K \\
2 r_{1} r_{2} x_{33}^{*} & =d_{12} K \\
r_{2}^{2} x_{33}^{*} & =d_{13} K \\
2 r_{1} r_{3} x_{33}^{*} & =d_{21} K \\
\left(2 r_{1} r_{4}+2 r_{2} r_{3}\right) x_{33}^{*} & =d_{22} K \\
2 r_{2} r_{4} x_{33}^{*} & =d_{23} K \\
r_{3}^{2} x_{33}^{*} & =d_{31} K \\
2 r_{3} r_{4} x_{33}^{*} & =d_{32} K \\
r_{4}^{2} x_{33}^{*}=d_{33} K & =K .
\end{aligned}
$$

Define $c_{1}, c_{2}>0$ by $c_{1}=x_{23}^{*} /\left(2 x_{33}^{*}\right)$ and $c_{2}=x_{32}^{*} /\left(2 x_{33}^{*}\right)$. Then $2 c_{1}=d_{23}$ and $2 c_{2}=d_{32}$. Moreover, (ii) $/(\mathrm{i}): 2 r_{2} / r_{1}=d_{12} / d_{11}$ and (ii) $/(\mathrm{iii}): 2 r_{1} / r_{2}=$ $d_{12} / d_{13}$ give $d_{12}^{2}=4 d_{11} d_{13}$. (viii)/(vii): $2 r_{4} / r_{3}=d_{32} / d_{31}$ and (viii)/(ix): $2 r_{3} / r_{4}=d_{32} / d_{33}=d_{32}$ give $d_{31}=c_{2}^{2}$. (iv) $/(\mathrm{i}): 2 r_{3} / r_{1}=d_{21} / d_{11}$ and (iv) $/($ vii): $2 r_{1} / r_{3}=d_{21} / d_{31}$ give $d_{21}=2 c_{2} \sqrt{d_{11}}$. (vi)/(iii): $2 r_{4} / r_{2}=d_{23} / d_{13}$ and (vi)/(ix): $2 r_{2} / r_{4}=d_{23} d_{33}=d_{23}$ give $d_{13}=c_{1}^{2}$. Thus, $d_{12}=2 c^{1} \sqrt{d_{11}}$. Since $r_{2} / r_{1}=c_{1} / \sqrt{d_{11}}, r_{3} / r_{1}=c_{2} / \sqrt{d_{11}}, r_{4} / r_{1}=1 / \sqrt{d_{11}}$, and $(\mathrm{v}) /(\mathrm{i}): 2 r_{4} / r_{1}+$ $2 r_{2} r_{3} / r_{1}^{2}=d_{22} / d_{11}$, therefore $d_{22}=2 c_{1} c_{2}+2 \sqrt{d_{11}}$. Substituting all of the above into the equation $x^{*}=K$, we get

$\left(d_{11}+2 c_{1} \sqrt{d_{11}}+c_{1}^{2}+2 c_{2} \sqrt{d_{11}}+2 c_{1} c_{2}+2 \sqrt{d_{11}}+2 c_{1}+c_{2}^{2}+2 c_{2}+1\right) x_{33}^{*}=K$

which in turn implies that $x_{33}^{*}=K /\left(\sqrt{d_{11}}+c_{1}+c_{2}+1\right)^{2}$. Now, substituting the latter into (i) and by noting $r_{1}=d_{11}+d_{12} / 2+d_{21} / 2+d_{22} / 4$, we have $d_{11}=c_{1}^{2} c_{1}^{2}$. From this, one can easily deduce that $X^{*}=H\left(c_{1}, c_{2}\right)$.

COROLlaRY 3.2. $C l\left(H\left(\stackrel{\circ}{+}_{+}^{2}\right)\right)=H\left(\mathbb{R}_{+}^{2}\right)$ is the set of equilibrium points of (NP) in $\mathbb{R}_{+}^{9} \backslash\{(0, \ldots, 0)\}$.

COROLLARY 3.3. $H\left(\mathbf{R}_{+}^{r}\right) \times\{0\}$ is the set of equilibrium points of (1.1) in $H_{x_{11}, \ldots, x_{33}} \backslash\{(0, \ldots, 0,0)\}$.

The main result of this section is a complete description of the global dynamics of (NP): all solutions $X(t)$ of (NP) other than $X(t) \equiv 0$ converge to the Hardy-Weinberg manifold as $t \rightarrow+\infty$. In order to describe this result more precisely and to present its proof, we need to introduce some auxiliary 
quantities. Define

$$
\begin{aligned}
& x_{A A}=x_{11}+x_{12}+x_{13} \\
& x_{A a}=x_{21}+x_{22}+x_{23} \\
& x_{a a}=x_{31}+x_{32}+x_{33}, \\
& x_{B B}=x_{11}+x_{21}+x_{31} \\
& x_{B b}=x_{12}+x_{22}+x_{32} \\
& x_{b b}=x_{13}+x_{23}+x_{33}, \\
& u_{A}=x_{A A}+x_{A a} / 2, \quad u_{a}=x_{a a}+x_{A a} / 2,
\end{aligned}
$$

and

$$
u_{B}=x_{B B}+x_{B b} / 2, \quad u_{b}=x_{b b}+x_{B b} / 2
$$

Clearly

$$
\begin{aligned}
& u_{A}+u_{a}=x_{A A}+x_{A a}+x_{a a}=x \\
& u_{B}+u_{b}=x_{B B}+x_{B b}+x_{b b}=x,
\end{aligned}
$$

and

$$
\begin{array}{ll}
u_{A}=u_{1}+u_{2}, & u_{a}=u_{3}+u_{4}, \\
u_{B}=u_{1}+u_{3}, & u_{b}=u_{2}+u_{4} .
\end{array}
$$

THEOREM 3.4. Define $c_{1}=u_{A}(0) / u_{a}(0)$ and $c_{2}=u_{B}(0) / u_{b}(0)$. Then

$$
\left(x_{11}(t), \ldots, x_{33}(t)\right) \rightarrow H\left(c_{1}, c_{2}\right)
$$

as $t \rightarrow+\infty$, where $H\left(c_{1}, c_{2}\right)$ was given in (3.4).

Before we prove Theorem 3.4, we need a couple of lemmas describing the long term behaviour of the auxiliary quantities defined in (3.9)-(3.12), as well as that of the $u_{i}$ 's.

LEMMA 3.5. Let $c_{1}=u_{A}(0) / u_{a}(0)$ and $c_{2}=u_{B}(0) / u_{b}(0)$. Then

$$
\begin{array}{ll}
\text { (I) } \quad u_{A}(t)=c_{1} u_{a}(t), \quad u_{B}(t)=c_{2} u_{b}(t) \quad \text { for all } t \geq 0 \\
\text { (II) } \quad\left(u_{A}(t), u_{a}(t)\right) \rightarrow\left(c_{1} K /\left(1+c_{1}\right), K /\left(1+c_{1}\right)\right) \\
& \left(u_{B}(t), u_{b}(t)\right) \rightarrow\left(c_{2} K /\left(1+c_{2}\right), K /\left(1+c_{2}\right)\right)
\end{array}
$$


as $t \rightarrow+\infty$, and,

$$
\text { (III) } \begin{aligned}
\left(x_{A A}(t), x_{A a}(t), x_{a a}(t)\right) \rightarrow\left(\frac{c_{1}^{2} K}{\left(1+c_{1}\right)^{2}}, \frac{2 c_{1} K}{\left(1+c_{2}\right)^{2}}, \frac{K}{\left(1+c_{1}\right)^{2}}\right) \\
\left(x_{B B}(t), x_{B b}(t), x_{b b}(t)\right) \rightarrow\left(\frac{c_{2}^{2} K}{\left(1+c_{2}\right)^{2}}, \frac{2 c_{2} K}{\left(1+c_{2}\right)^{2}}, \frac{K}{\left(1+c_{2}\right)^{2}}\right) \\
\text { as } t \rightarrow+\infty .
\end{aligned}
$$

Proof. Clearly

$$
\begin{aligned}
\dot{x}_{A A} & =\left(u_{A}^{2} / x^{2}\right) B(x)-\left(x_{A A} / x\right) D(x) \\
\dot{x}_{A a} & =\left(2 u_{A} u_{a} / x^{2}\right) B(x)-\left(x_{A a} / x\right) D(x) \\
\dot{x}_{a a} & =\left(u_{a}^{2} / x^{2}\right) B(x)-\left(x_{a a} / x\right) D(x)
\end{aligned}
$$

and

$$
\begin{aligned}
\dot{x}_{B B} & =\left(u_{B}^{2} / x^{2}\right) B(x)-\left(x_{B B} / x\right) D(x) \\
\dot{x}_{B b} & =\left(2 u_{B} u_{b} / x^{2}\right) B(x)-\left(x_{B b} / x\right) D(x) \\
\dot{x}_{b b} & =\left(u_{b}^{2} / x^{2}\right) B(x)-\left(x_{b b} / x\right) D(x) .
\end{aligned}
$$

Proceeding as in the one-locus case (c.f. p. 369 of Freedman and Waltman [5], and p. 231 of So [9]) one can easily show that (I) holds and that

$$
\begin{aligned}
& x_{A A}(t)-c_{1}^{2} x_{a a}(t) \rightarrow 0, \quad x_{A a}(t)-2 c_{1} x_{a a}(t) \rightarrow 0 \\
& x_{B B}(t)-c_{2}^{2} x_{b b}(t) \rightarrow 0, \quad x_{B b}(t)-2 c_{2} x_{b b}(t) \rightarrow 0,
\end{aligned}
$$

as $t \rightarrow+\infty$. (II) and (III) now follow from (3.2), (3.13) and (3.23).

REMARK. Lemma 3.5 and (3.14) show that

$$
\begin{gathered}
u_{1}(t)=c_{2} u_{b}(t)-u_{a}(t)+u_{4}(t)=c_{1} u_{a}(t)-u_{b}(t)+u_{4}(t) \\
u_{2}(t)=u_{b}(t)-u_{4}(t), \quad u_{3}(t)=u_{a}(t)-u_{4}(t)
\end{gathered}
$$

for all $t \geq 0$, where $c_{1}=u_{A}(0) / u_{a}(0)$ and $c_{2}=u_{B}(0) / u_{b}(0)$.

LEMMA 3.6. Let $c_{1}=u_{A}(0) / u_{a}(0)$ and $c_{2}=u_{B}(0) / u_{b}(0)$. Then

$$
\begin{aligned}
& \left(u_{1}(t), u_{2}(t), u_{3}(t), u_{4}(t)\right) \rightarrow \\
& \quad\left(\frac{c_{1} c_{2} K}{\left(1+c_{1}\right)\left(1+c_{2}\right)}, \frac{c_{1} K}{\left(1+c_{1}\right)\left(1+c_{2}\right)}, \frac{c_{2} K}{\left(1+c_{1}\right)\left(1+c_{2}\right)}, \frac{K}{\left(1+c_{1}\right)\left(1+c_{2}\right)}\right)
\end{aligned}
$$

as $t \rightarrow \infty$. 
Proof. The proof is divided into three steps.

Step 1: $u_{i}(i=1,2,3,4)$ satisfy the following system of differential equations.

$$
\begin{aligned}
& \dot{u}_{1}=\left(u_{1}^{2}+u_{1} u_{2}+u_{1} u_{3}+\frac{1}{2} u_{1} u_{4}+\frac{1}{2} u_{2} u_{3}\right) \frac{B(x)}{x^{2}}-u_{1} \frac{D(x)}{x} \\
& \dot{u}_{2}=\left(u_{2}^{2}+u_{1} u_{2}+u_{2} u_{4}+\frac{1}{2} u_{1} u_{4}+\frac{1}{2} u_{2} u_{3}\right) \frac{B(x)}{x^{2}}-u_{2} \frac{D(x)}{x} \\
& \dot{u}_{3}=\left(u_{3}^{2}+u_{1} u_{3}+u_{3} u_{4}+\frac{1}{2} u_{1} u_{4}+\frac{1}{2} u_{2} u_{3}\right) \frac{B(x)}{x^{2}}-u_{3} \frac{D(x)}{x} \\
& \dot{u}_{4}=\left(u_{4}^{2}+u_{2} u_{4}+u_{3} u_{4}+\frac{1}{2} u_{1} u_{4}+\frac{1}{2} u_{2} u_{3}\right) \frac{B(x)}{x^{2}}-u_{4} \frac{D(x)}{x}
\end{aligned}
$$

where $x=u_{1}+u_{2}+u_{3}+u_{4}$. This follows directly from (NP), (1.2) and (1.3). Step 2:

$$
\begin{aligned}
& u_{1}(t)-c_{2} u_{2}(t) \\
& =\left(u_{1}(0)-c_{1} u_{2}(0)\right) \exp \left[\int_{0}^{t}\left(\frac{\left(1+c_{1}\right) u_{a}(\tau) B(x(\tau))}{2 x^{2}(\tau)}-\frac{D(x(\tau))}{x(\tau)}\right) d \tau\right] \\
& u_{3}(t)-c_{2} u_{4}(t) \\
& =\left(u_{3}(0)-c_{2} u_{4}(0)\right) \exp \left[\int_{0}^{t}\left(\frac{\left(1+c_{1}\right) u_{a}(\tau) B(x(\tau))}{2 x^{2}(\tau)}-\frac{D(x(\tau))}{x(\tau)}\right) d \tau\right] \\
& u_{1}(t)-c_{1} u_{3}(t) \\
& =\left(u_{1}(0)-c_{1} u_{3}(0)\right) \exp \left[\int_{0}^{t}\left(\frac{\left(1+c_{1}\right) u_{a}(\tau) B(x(\tau))}{2 x^{2}(\tau)}-\frac{D(x(\tau))}{x(\tau)}\right) d \tau\right] \\
& u_{2}(t)-c_{1} u_{4}(t) \\
& =\left(u_{2}(0)-c_{1} u_{4}(0)\right) \exp \left[\int_{0}^{t}\left(\frac{\left(1+c_{1}\right) u_{a}(\tau) B(x(\tau))}{2 x^{2}(\tau)}-\frac{D(x(\tau))}{x(\tau)}\right) d \tau\right] .
\end{aligned}
$$

This can be proved by writing down the linear differential equation that each of the functions on the LHS of (3.27) satisfies. For example, by (3.24) and (3.26)

$$
\begin{aligned}
\dot{u}_{2}-c_{1} \dot{u}_{4} & =\left(u_{2}-c_{1} u_{4}\right)\left[\left(1+c_{2}\right) u_{b} B(x) /\left(2 x^{2}\right)-D(x) / x\right] \\
& =\left(u_{2}-c_{1} u_{4}\right)\left[\left(1+c_{1}\right) u_{a} B(x) /\left(2 x^{2}\right)-D(x) / x\right]
\end{aligned}
$$

Step 3:

$$
\begin{array}{ll}
u_{1}(t)-c_{2} u_{2}(t) \rightarrow 0, & u_{3}(t)-c_{2} u_{4}(t) \rightarrow 0, \\
u_{1}(t)-c_{1} u_{3}(t) \rightarrow 0, & u_{2}(t)-c_{1} u_{4}(t) \rightarrow 0,
\end{array}
$$

as $t \rightarrow+\infty$. This can be proved by noting that (3.2), (3.7) and (3.18) imply

$$
\frac{\left(1+c_{1}\right) u_{a}(t) B(x(t))}{2 x^{2}(t)}-\frac{D(x(t))}{x(t)} \rightarrow-\frac{B(K)}{2 K}<0
$$


as $t \rightarrow \infty$. Therefore, the integral in (3.27) is divergent and consequently we have (3.28).

Using (3.28) and (1.3), it is then easy to see that (3.25) holds.

Proof of Theorem 3.4. To show (3.15), it suffices to show

$$
\begin{aligned}
x_{11}(t)-c_{1}^{2} c_{2}^{2} x_{33}(t) & \rightarrow 0, x_{12}(t)-2 c_{1}^{2} c_{2} x_{33}(t) \rightarrow 0, x_{13}(t)-c_{1}^{2} x_{33}(t) \rightarrow 0, \\
x_{21}(t)-2 c_{1} c_{2}^{2} x_{33}(t) & \rightarrow 0, x_{22}(t)-4 c_{1} c_{2} x_{33}(t) \rightarrow 0, x_{23}(t)-2 c_{1} x_{33}(t) \rightarrow 0, \\
x_{31}(t)-c_{2}^{2} x_{33}(t) & \rightarrow 0, x_{32}(t)-2 c_{2} x_{33}(t) \rightarrow 0
\end{aligned}
$$

as $t \rightarrow+\infty$. We illustrate this by showing $z(t) \equiv x_{32}(t)-2 c_{2} x_{33}(t) \rightarrow 0$ as $t \rightarrow+\infty$. Clearly $z(t)$ satisfies the linear differential equation

$$
\dot{z}(t)+Q_{1}(t) z(t)=Q_{2}(t)
$$

where $Q_{1}(t)=D(x(t)) / x(t), Q_{2}(t)=2 u_{4}(t)\left[u_{3}(t)-c_{2} u_{4}(t)\right] B(x(t)) / x^{2}(t)$. Solving this equation yields

$$
z(t)=z(0) \exp \left[-\int_{0}^{t} Q_{1}(\tau) d \tau\right]+\int_{0}^{t} \exp \left[-\int_{s}^{t} Q_{1}(\tau) d \tau\right] Q_{2}(s) d s .
$$

We shall show that both the first and the second term tend to 0 as $t$ tends to $+\infty$

Since $\lim _{t \rightarrow+\infty} Q_{1}(t)=D(K) / K$, by (3.2), $\int_{0}^{+\infty} Q_{1}(t) d t=+\infty$, and hence, $\lim _{t \rightarrow+\infty} \exp \left[-\int_{0}^{t} Q_{1}(\tau) d \tau\right]=0$. On the other hand, by (3.27),

$$
\begin{aligned}
\int_{0}^{t} \exp & {\left[-\int_{s}^{t} Q_{1}(\tau) d \tau\right] Q_{2}(s) d s } \\
= & 2\left(u_{3}(0)-c_{2} u_{4}(0)\right) \exp \left[-\int_{0}^{t} \frac{D(x(\tau))}{x(\tau)} d \tau\right] \\
& \cdot \int_{0}^{t} u_{4}(s) \exp \left[\frac{1+c_{1}}{2} \int_{0}^{s} \frac{u_{a}(\tau) B(x(\tau))}{x^{2}(\tau)} d \tau\right] \frac{B(x(s))}{x^{2}(s)} d s .
\end{aligned}
$$

To show that the LHS of (3.29) tends to 0 as $t$ tends to $+\infty$, it suffices, by L'Hospital's rule, to show that

$$
\lim _{t \rightarrow+\infty} \frac{u_{4}(t) \exp \left[\frac{1+c_{1}}{2} \int_{0}^{t} \frac{u_{a}(\tau) B(x(\tau))}{x^{2}(\tau)} d \tau\right] \frac{B(x(t))}{x^{2}(t)}}{\exp \left[\int_{0}^{t} \frac{D(x(\tau))}{x(\tau)} d \tau\right] \frac{D(x(t))}{x(t)}}=0 .
$$

Since $\lim _{t \rightarrow+\infty} u_{4}(t)=K /\left(1+c_{1}\right)\left(1+c_{2}\right)>0, \lim _{t \rightarrow+\infty} B(x(t)) / x(t)=$ $B(K) / K>0$, and, $\lim _{t \rightarrow+\infty} D(x(t)) / x(t)=D(K) / K>0$, it suffices to show

$$
\lim _{t \rightarrow+\infty} \int_{0}^{t}\left[\frac{D(x(\tau))}{x(\tau)}-\frac{\left(1+c_{1}\right) u_{a}(\tau) B(x(\tau))}{2 x^{2}(\tau)}\right] d \tau=+\infty
$$


But this follows from

$$
\begin{aligned}
\lim _{t \rightarrow+\infty}\left[\frac{D(x(t))}{x(t)}-\frac{\left(1+c_{1}\right) u_{a}(t) B(x(t))}{2 x^{2}(t)}\right] & =\frac{D(K)}{K}-\frac{1+c_{1}}{2} \cdot \frac{K}{1+c_{1}} \cdot \frac{B(K)}{K^{2}} \\
& =\frac{B(K)}{2 K}>0 .
\end{aligned}
$$

In the following corollary we reformulate Theorem 3.4 in terms of proportions.

COROLlaRY 3.7. If $\left(x_{11}(t), \ldots, x_{33}(t)\right)$ is a solution of (NP) other than the trivial solution $(0, \ldots, 0)$, then

$$
\begin{aligned}
x_{11}(t) & : x_{12}(t): x_{13}(t): x_{21}(t): x_{22}(t): x_{23}(t): x_{31}(t): x_{32}(t): x_{33}(t) \\
& \rightarrow c_{1}^{2} c_{2}^{2}: 2 c_{1}^{2} c_{2}: c_{1}^{2}: 2 c_{1} c_{2}^{2}: 4 c_{1} c_{2}: 2 c_{1}: c_{2}^{2}: 2 c_{2}: 1
\end{aligned}
$$

as $t \rightarrow+\infty$, where

$$
c_{1}=\frac{x_{11}(0)+x_{12}(0)+x_{13}(0)+\frac{1}{2} x_{21}(0)+\frac{1}{2} x_{22}(0)+\frac{1}{2} x_{23}(0)}{x_{31}(0)+x_{32}(0)+x_{33}(0)+\frac{1}{2} x_{21}(0)+\frac{1}{2} x_{22}(0)+\frac{1}{2} x_{23}(0)}
$$

and

$$
c_{2}=\frac{x_{11}(0)+x_{21}(0)+x_{31}(0)+\frac{1}{2} x_{12}(0)+\frac{1}{2} x_{22}(0)+\frac{1}{2} x_{32}(0)}{x_{13}(0)+x_{23}(0)+x_{33}(0)+\frac{1}{2} x_{12}(0)+\frac{1}{2} x_{22}(0)+\frac{1}{2} x_{32}(0)}
$$

\section{Conditions which lead to evolution of pure strains}

In this section, conditions leading to the global stability of one of the boundary equilibrium points $E_{i}(i=1,2,3,4)$ will be considered. First we describe a condition which guarantees the persistence of $y$.

Proposition 4.1. Let

$$
\begin{aligned}
d\left(c_{1}, c_{2}\right)= & -s+\frac{K}{\left(1+c_{1}\right)^{2}\left(1+c_{2}\right)^{2}}\left[c_{1}^{2} c_{2}^{2} P_{11}(K)+2 c_{1}^{2} c_{2} P_{12}(K)\right. \\
& +c_{1}^{2} P_{13}(K)+2 c_{1} c_{2}^{2} P_{21}(K)+4 c_{1} c_{2} P_{22}(K)+2 c_{1} P_{23}(K) \\
& \left.+c_{2}^{2} P_{31}(K)+c_{2} P_{32}(K)+P_{33}(K)\right] .
\end{aligned}
$$

If there exists $\zeta>0$ such that

$$
d\left(c_{1}, c_{1}\right) \geq \zeta \quad \text { for all } c_{1}, c_{2} \geq 0,
$$

then $y$ is uniformly persistent, that is, there exists $\eta>0$ such that $\underline{\lim }_{t \rightarrow+\infty} y(t)$ $\geq \eta$ for all $\left(x_{11}(0), \ldots, x_{33}(0), y(0)\right) \in \stackrel{\circ}{+}_{+}^{10}$. 
Proof. Let $\left(x_{11}, \ldots, x_{33}, y\right) \in \stackrel{\circ}{\mathbf{R}}_{+}^{10}$. Since the acyclic condition is satisfied on $H_{x_{11}, \ldots, x_{33}}$, by Butler, Freedman and Waltman [2, Section 3], it suffices to show that $y$ is persistent. We note that $d\left(c_{1}, c_{2}\right)$ is the eigenvalue of the variational matrix, $M\left(E_{c_{1}, c_{2}}\right)$, of (1.1) evaluated at the equilibrium point $E_{c_{1}, c_{2}}=\left(H\left(c_{1}, c_{2}\right), 0\right)$ corresponding to eigenvectors of $M\left(E_{c_{1}, c_{2}}\right)$ which have a nonzero $y$ component. Let $\varepsilon$ be the set of all equilibrium points of (1.1) in $H_{x_{11}, \ldots, x_{33}} \backslash\{(0, \ldots, 0,0)\}$, that is, $\varepsilon=\left\{E_{c_{1}, c_{2}}: c_{1}, c_{2} \geq 0\right\}$. Then by center manifold theory, $\varepsilon$ is an isolated invariant set. According to hypothesis (4.2), the stable set, $W^{s}(\varepsilon)$, of $\varepsilon$ has an empty intersection with $\stackrel{\circ}{+}_{+}^{10}$. By Theorem 4.1 of Butler and Waltman [1], in order for $\underline{\lim }_{t \rightarrow+\infty} y(t)=0$, the omega-limit set, $\omega\left(x_{11}(0), \ldots, x_{33}(0), y(0)\right)$, of the point $\left(x_{11}(0), \ldots, x_{33}(0), y(0)\right)$ must intersect $W^{s}(\varepsilon)$ at a point other than those in $\varepsilon$. Now $W^{s}(\varepsilon) \cap \mathbf{R}_{+}^{10} \subset H_{x_{11}, \ldots, x_{33}}$, with the global dynamics on $H_{x_{11}, \ldots, x_{33}}$ as given in Theorem 3.4, implies that $\omega\left(x_{11}(0), \ldots, x_{33}(0), y(0)\right)$ either contains $E_{0}$ or is unbounded. In either case, we have a contradiction (cf. Proposition 2.1).

Since the analysis for the rest of this section and the next requires a knowledge of the global dynamics of (1.1) on the boundary positively invariant set $\nu$, we will make the following additional assumptions.

(A1) The predator $y$ persists. (By Proposition 4.1, it suffices to assume that $d\left(c_{1}, c_{2}\right) \geq \zeta>0$ for all $\left.c_{1}, c_{2} \geq 0\right)$.

(A2) $E_{1}$ (resp. $E_{2}, E_{3}, E_{4}$ ) exists and is globally exponentially stable on $\stackrel{\circ}{H}_{x_{11, y}}$, the positive $x_{11}-y$ cone (resp. $\stackrel{\circ}{H}_{x_{13, y}}, \stackrel{\circ}{H}_{x_{31, y}}, \stackrel{\circ}{H}_{x_{33, y}}$ ). (Some sufficient conditions for the global stability of a positive equilibrium point of generalised Gauss predator-prey systems were given in Hsu [8] and in Cheng, Hsu and Lin [3].)

(A3) $E_{i}(i=1,2,3,4)$ are hyperbolic. (By looking at $M\left(E_{i}\right)$ (c.f. Table 1), one can easily show that it is equivalent to assume

$$
\left.\bar{y}_{i j}\left[s / k-P_{22}\left(\bar{x}_{i j}\right)\right] \neq B\left(\bar{x}_{i j}\right) / 2 \text { for }(i, j=1,3) .\right)
$$

The following theorem provides conditions under which the equilib-

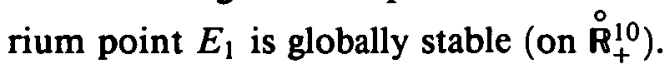

TheOREM 4.2. Suppose (H1)-(H6) and (A1)-(A3) hold. If the $P_{i j}(i, j=$ $1,2,3)$ are totally ordered:

$$
P_{11} \triangleleft P_{12} \triangleleft P_{13} \triangleleft P_{21} \triangleleft P_{22} \triangleleft P_{23} \triangleleft P_{31} \triangleleft P_{32} \triangleleft P_{33}
$$

where $P_{l j} \triangleleft P_{h l}$ means that there exists $e>0$ such that $P_{h l}(x)-P_{i j}(x) \geq e x$ for all $x \in[0, K]$, then $E_{1}$ is globally stable on $\stackrel{\mathbf{R}}{+}_{+}^{10}$. 
Proof. Let $\left(x_{11}, \ldots, x_{33}, y\right) \in \stackrel{\circ}{\mathbf{R}}_{+}^{10}$. Denote the omega limit set of $\left(x_{11}, \ldots\right.$, $\left.x_{33}, y\right)$ by $\omega \equiv \omega\left(x_{11}, \ldots, x_{33}, y\right)$. The proof proceeds in two steps. In step 1 , we will show that $\omega \subset \partial\left(\mathbf{R}_{+}^{10}\right)$ and in step 2, we show that no point in $\partial\left(\mathbf{R}_{+}^{10}\right)$ can lie in $\omega$ except $E_{1}$. Therefore, $E_{1} \in \omega$. Now, by assumption, $E_{1}$ is asymptotically stable and thus $\omega=\left\{E_{1}\right\}$. Hence, $E_{1}$ is globally stable.

Step 1. Using REDUCE (an algebraic manipulation computer language), one shows that

$$
\frac{\dot{u}_{a}}{u_{a}}-\frac{\dot{u}_{A}}{u_{A}} \leq-\frac{y}{u_{A} u_{a}}\left[e_{33} x_{13} x_{33}+\text { non-negative terms }\right],
$$

where $e_{33}$ is a positive constant. Therefore as in the proof of Theorem 3.1 in Freedman, So and Waltman [7].

$$
u_{a}(t) \leq \frac{u_{a}(0)}{u_{A}(0)} u_{A}(t) \exp \left[-\frac{e_{33}}{K^{2}} \int_{0}^{t}\left(x_{12} x_{33} y\right)(\tau) d \tau\right] .
$$

If the integral diverges, then $\lim _{t \rightarrow+\infty} u_{a}(t)=0$ so that $\omega \cap \partial\left(\mathbf{R}_{+}^{10}\right) \neq \varnothing$. Otherwise, $z(t) \equiv\left(x_{12} x_{33} y\right)(t) \in L^{1}[0,+\infty)$. Since $\dot{z}(t)$ is bounded, we have, $\lim _{t \rightarrow+\infty} z(t)=0$. Hence, $\underline{\lim }_{t \rightarrow+\infty} x_{12}(t)=0$ or $\underline{\lim }_{t \rightarrow+\infty} x_{33}(t)=0$ (since $\underline{\lim }_{t \rightarrow+\infty} y(t)>0$ by (A1)). This again implies $\omega \cap \partial\left(R_{+}^{10}\right) \neq \varnothing$.

Step 2. It was pointed out in Section 2 that no point in $\partial\left(R_{+}^{10}\right) \backslash \nu$ can lie in $\omega$. Therefore, it suffices to consider only points in $\nu$. By looking at the eigenvalues of the variational matrix $M\left(E_{i}\right)(i=1,2,3,4)$ (cf. Table 1), we see that the dimension $(\operatorname{dim})$ of the unstable manifolds, $W^{u}\left(E_{i}\right)$, of $E_{i}$ $(i=1,2,3,4)$ satisfy $\operatorname{dim} W^{u}\left(E_{1}\right)=0, \operatorname{dim} W^{u}\left(E_{2}\right) \geq 1, \operatorname{dim} W^{u}\left(E_{3}\right) \geq 1$ and $\operatorname{dim} W^{u}\left(E_{4}\right) \geq 2$. Therefore $E_{1}$ is asymptotically stable and $W^{u}\left(E_{i}\right)$ $(i=2,3,4)$ have a nonempty intersection with $\mathbb{R}^{10} \backslash \mathbb{R}_{+}^{10}$. Consequently, by Proposition 2.2, we see that $W^{s}\left(E_{i}\right) \cap \mathbf{R}_{+}^{10} \subset \partial\left(\mathbf{R}_{+}^{10}\right)$. On the other hand the main result for the one-locus model (cf. Theorem 3.1 of Freedman, So and Waltman [7]) tells us that $E_{2}$ (resp. $E_{3}, E_{4}$ ) is globally stable on $\stackrel{\circ}{H}_{x_{13}, x_{23}, x_{33}, y}$ (resp. $\stackrel{\circ}{H}_{x_{31}, x_{32}, x_{33}, y}, \stackrel{\circ}{H}_{x_{33, y}}$ ). Thus, $W^{s}\left(E_{2}\right) \cap \mathbf{R}_{+}^{10} \subset H_{x_{13}, x_{23}, x_{33}, y}, W^{s}\left(E_{3}\right) \cap \mathbf{R}_{+}^{10} \subset$ $H_{x_{31}, x_{32}, x_{33}, y}$ and $W^{s}\left(E_{4}\right) \cap \mathbf{R}_{+}^{10} \subset H_{x_{33}, y}$. Knowing the global dynamics on $\nu$, it follows easily (using Theorem 4.1 of Butler and Waltman [1]) that $E_{1}$ is the only point in $\partial\left(\mathbf{R}_{+}^{10}\right)$ that can lie in $\omega$.

\section{Some persistence results}

In this section we consider conditions which lead to the uniform persistence of system (1.1). Due to the large number of possibilities, we shall restrict ourselves to the cases when Theorem 3.1 of Freedman, So and Waltman [7] applies; that is, the predator functional responses for the heterozygotes 
are ordered between the homozygote ones. Under this restriction, the global dynamics of (1.1) on the boundary invariant set $\nu$ is known. By interchanging the roles of the allele type $A$ and $a$, or $B$ and $b$ if necessary, there are three cases to consider.

CASE 1: $P_{11} \triangleleft P_{12} \triangleleft P_{13}, \quad P_{11} \triangleleft P_{21} \triangleleft P_{31}, \quad P_{13} \triangleleft P_{23} \triangleleft P_{33}, \quad P_{31} \triangleleft P_{32} \triangleleft P_{33}$.

CASE 2: $P_{11} \triangleleft P_{12} \triangleleft P_{13}, \quad P_{11} \triangleleft P_{21} \triangleleft P_{31}, \quad P_{33} \triangleleft P_{23} \triangleleft P_{13}, \quad P_{33} \triangleleft P_{32} \triangleleft P_{31}$.

CASE 3: $P_{11} \triangleleft P_{12} \triangleleft P_{13}, \quad P_{11} \triangleleft P_{21} \triangleleft P_{31}, \quad P_{13} \triangleleft P_{23} \triangleleft P_{33}, \quad P_{33} \triangleleft P_{32} \triangleleft P_{31}$.

THEOREM 5.1. Under the assumptions (H1)-(H6) and (A1)-(A3), system (1.1) is uniformly persistent if

(i) $\bar{y}_{11}\left(s / k-P_{22}\left(\bar{x}_{11}\right)\right)-B\left(\bar{x}_{11}\right) / 2>0$ under Case 1 and Case 3, and

(ii) $\bar{y}_{i i}\left(s / k-P_{22}\left(\bar{x}_{i i}\right)\right)-B\left(\bar{x}_{i i}\right) / 2>0, i=1,3$ under Case 2.

Proof. We only discuss Case 1 . The other two cases are similar. Let $\Omega=c l\left(\cup_{p \in \nu} \omega(p)\right)$ be the global attractor in $\nu$. Then $\left\{\mathscr{M}_{i}\right\}_{i=0}^{5}$, where $\mathscr{M}_{i}=$ $\left\{E_{i}\right\},(i=1, \ldots, 5)$ and $\mathscr{M}_{5}=\varepsilon$, is an acyclic isolated covering of $\Omega$. According to Theorem 3.1 of Butler and Waltman [1], it suffices to show that $W^{s}\left(\mathscr{M}_{i}\right) \cap \stackrel{\circ}{\mathrm{R}}_{+}^{10}=\varnothing(i=1, \ldots, 5)$. Firstly, $W^{s}\left(\mathscr{M}_{5}\right) \cap \stackrel{\circ}{\mathbf{R}}_{+}^{10}=\varnothing$ follows from (A1). Secondly, $\operatorname{dim} W^{u}\left(E_{4}\right) \geq 2$ implies $W^{u}\left(E_{4}\right) \cap\left(\mathbf{R}^{10} \backslash \stackrel{\circ}{+}_{+}^{10}\right) \neq \varnothing$ and hence by Proposition $2.2, W^{s}\left(E_{4}\right) \cap \stackrel{\circ}{\mathbf{R}}_{+}^{10}=W^{s}\left(\mathscr{M}_{4}\right) \cap \stackrel{\circ}{\mathbf{R}}_{+}^{10}=\varnothing$. Similarly, one can show that $\left.W^{s}\left(\mathscr{M}_{i}\right) \cap \stackrel{\circ}{\mathrm{R}}_{+}^{10}=\varnothing\right)(i=1,2,3)$, since $\operatorname{dim} W^{u}\left(E_{i}\right) \geq 1(i=1,2,3)$. Finally, $W^{s}\left(\mathscr{M}_{0}\right) \cap \stackrel{\circ}{\mathbf{R}}_{+}^{10}=W^{s}\left(E^{0}\right) \cap \stackrel{\circ}{\mathbf{R}}_{+}^{10}=\varnothing$ follows from Proposition 2.1.

\section{Discussion}

In Section 4, we demonstrated one case where all the prey allele types in system (1.1) become extinct except one. This is analogous to the previously studied one-locus model. In contrast to that, we also illustrated in Section 5 that there are cases for which, when considered as a one-locus problem, some prey allele types becomes extinct, whereas by making the predator functional 
response $P_{22}$ for the double heterozygote sufficiently small, we get persistence for all the prey allele types (as well as the predator).

\section{Acknowledgement}

This research was partially supported by the University of Alberta Central Research Fund and the Natural Sciences and Engineering Research Council of Canada, grant number NSERC OGP36475.

\section{References}

[1] G. J. Butler and P. E. Waltman, "Persistence in dynamical systems", J. Differential Equations, 63 (1986) 255-263.

[2] G. J. Butler, H. I. Freedman and P. E. Waltman, "Uniformly persistent systems", Proc. Amer. Math. Soc., 96 (1986) 425-430.

[3] K.-S. Cheng, S.-B. Hsu and S.-S. Lin, "Some results on global stability of a predator-prey system", J. Math. Biol., 12 (1981) 115-126.

[4] H. I. Freedman, Deterministic mathematical models in population ecology (Marcel Dekker, New York, 1980).

[5] H. I. Freedman and P. E. Waltman, "Predator influence on the growth of a population with three genotypes", J. Math. Biol., 6 (1978) 367-374.

[6] H. I. Freedman and P. E. Waltman, "Predator influence on the growth of a population with three genotypes II", Rocky Mountain J. Math., 12 (1982) 779-784.

[7] H. I. Freedman, J. W.-H. So and P. E. Waltman, "Predatory influence on the growth of a population with three genotypes III", J. Math. Anal. Appl., 128 (1987) 287-304.

[8] S. B. Hsu, "On the global stability of a predator-prey system", Math. Biosci., 39 (1978) $1-10$.

[9] J. W.-H. So, “Analysis of a continuous one-locus, two-allele genetic model with genotypic fertility differences", Math. Biosci., 82 (1986) 221-233.

[10] J. W.-H. So and H. I. Freedman, "Persistence and global stability in a predatory-prey model consisting of three prey genotypes with fertility differences", Bull. Math. Biol., 48 (1986) 469-484. 